\title{
Electron-Electron Interaction Effects in Quantum Hall Regime of GaN/AlGaN Heterostructures
}

\author{
M. Siekacz ${ }^{a, *}$ K. Dybko ${ }^{b}$, D. Maude $^{c}$, M. Potemski $^{c}$, \\ W. KNAP ${ }^{d}$ AND C. SKIERBISZEWSKI ${ }^{a}$ \\ ${ }^{a}$ High Pressure Research Center, Polish Academy of Sciences \\ Sokołowska 29/37, 01-142 Warsaw, Poland \\ ${ }^{b}$ Institute of Physics, Polish Academy of Sciences \\ al. Lotników 32/46, 02-668 Warsaw, Poland
}

${ }^{c}$ Grenoble High Magnetic Field Laboratory, MPI-CNRS, 38042 Grenoble, France

${ }^{d}$ GES-UMR, CNRS-Université Montpellier 2

Place E. Bataillon, 34950 Montpellier, France

\begin{abstract}
We measured the activation of resistivity at quantum Hall minima in high mobility two-dimensional electron gas confined at AlGaN/GaN interface. The effective $g$-factor and effective mass was deduced. The electronelectron interactions modify both quantities compared to their bare band values. It is found that the influence of interactions is much more pronounced onto $g$-factor than effective mass. The relative spin susceptibility was also calculated and compared with available theories. The best agreement was found with the ideal two-dimensional gas model in random phase approximation.
\end{abstract}

PACS numbers: 71.30.+h, 73.40.-c, 73.43.Qt

\section{Introduction}

The electron effective mass, $m^{*}$, and effective $g$-factor, $g^{*}$, values can be determined in quantum Hall regime from activation energies obtained at even and odd filling factors, respectively. However, the $m^{*}$ and $g^{*}$ inferred in such a procedure are renormalized due to the electron-electron interactions. The magnitude of this renormalization depends on the ratio of the Coulomb interaction energy to the Fermi energy. It is characterized by the dimensionless Wigner-Seitz radius:

*corresponding author; e-mail: msiekacz@unipress.waw.pl 
$r_{\mathrm{s}}=(\pi n)^{-1 / 2} / a_{\mathrm{B}}$ where $n$ is the electron density, $a_{\mathrm{B}}=\epsilon^{2} / e^{2} m_{0}$ is the effective Bohr radius, $\epsilon$ - the static dielectric constant, and $m_{0}$ - the effective mass without interactions included [1]. Thus, the system is defined as strongly interacting at large $r_{\mathrm{s}}$ and weakly interacting at small $r_{\mathrm{s}}\left(r_{\mathrm{s}} \approx 1\right)$. Since electron-electron interaction influences both $m^{*}$ and $g^{*}$, it is useful to analyze the value which is proportional to its product - the spin susceptibility $\chi^{*}\left(\chi^{*} \propto g^{*} m^{*}\right)$. The increase in the spin susceptibility with interaction strength has been already experimentally confirmed in different two-dimensional systems (2DS) (Si-MOSFETs, GaAs/AlGaAs heterostructures, AlAs, and InGaAs/InAlAs) [1, 2].

In this contribution we present the spin susceptibility data for GaN/AlGaN heterostructures grown by plasma assisted MBE on dislocation free bulk GaN substrates. The growth technique together with high quality substrates allowed us to achieve very high electron mobilities of 2DS. From activation energies obtained at odd and even filling factors the effective $g$-factor and effective mass are evaluated and compared to their bare values. The resulting spin susceptibility is compared with the latest theoretical predictions.

\section{Samples and experimental results}

We have grown GaN/AlGaN structures by plasma assisted MBE on low dislocation density GaN crystals (on Ga polarity side) [3]. Substrates for growth were prepared by mechano-polishing procedure. The samples consist of a $1 \mu \mathrm{m}$ undoped GaN layer which is followed by $250 \mathrm{~nm}$ of undoped AlGaN barrier and finally covered by $3 \mathrm{~nm}$ GaN cap layer. We have chosen two samples of electron concentration at liquid helium temperature equal to $2.1 \times 10^{12} \mathrm{~cm}^{-2}$ and $5.6 \times 10^{11} \mathrm{~cm}^{-2}$. These electron densities translate into $r_{\mathrm{s}}=1.75$ and $r_{\mathrm{s}}=3.3$, respectively, which in turn correspond to the regime of intermediate strength of electron-electron interactions. The high electron mobility $\left(60000 \mathrm{~cm}^{2} /(\mathrm{V} \mathrm{s})\right.$ and $\left.20000 \mathrm{~cm}^{2} /(\mathrm{V} \mathrm{s})\right)$ allowed us to study the integer quantum Hall effect in a temperature range from $1.5 \mathrm{~K}$ to $30 \mathrm{~K}$ in high magnetic fields given by the Bitter magnet swept up to $23 \mathrm{~T}$. The measurements were done by a standard low frequency lock-in technique supplying the samples with the current of the order of $100 \mathrm{nA}$. The typical temperature dependences of the longitudinal magnetoresistance in the integer quantum Hall regime are presented in Fig. 1a, while in Fig. 1b the Arrhenius plots of the resistivities taken at different filling factors are shown. The data in Fig. 1b exhibit clearly the straight line regions - which can be easily used for determination of activation energies. However, we would like to point out limits of this approach - at high temperatures, when the corresponding $R_{x x}$ minimum is poorly resolved, the transport is no longer thermally activated. Also the low temperature extreme of several data exhibits some curvature, which is conventionally attributed to the onset of hopping over localized states.

When the Fermi level is located between two consecutive discrete Landau levels separated by the energy gap $\Delta E$, which satisfies $\Delta E \gg k_{\mathrm{B}} T$, the resistivity 

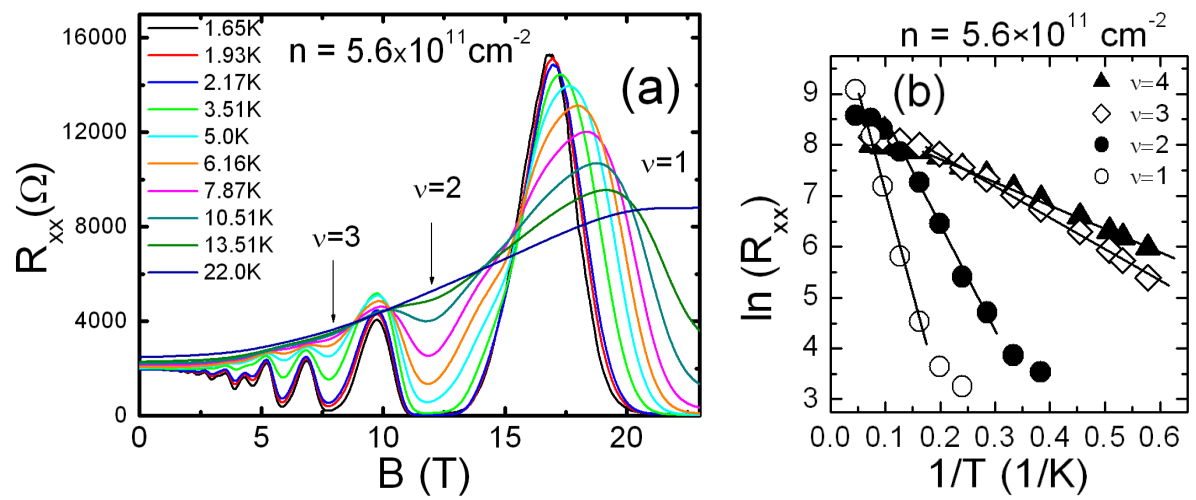

Fig. 1. The temperature dependence of the longitudinal magnetoresistance in the integer quantum Hall regime as measured for lower density sample $n=5.6 \times 10^{11} \mathrm{~cm}^{-2}$ (a). The Arrhenius plots of activated resistivity at different filling factors (b).
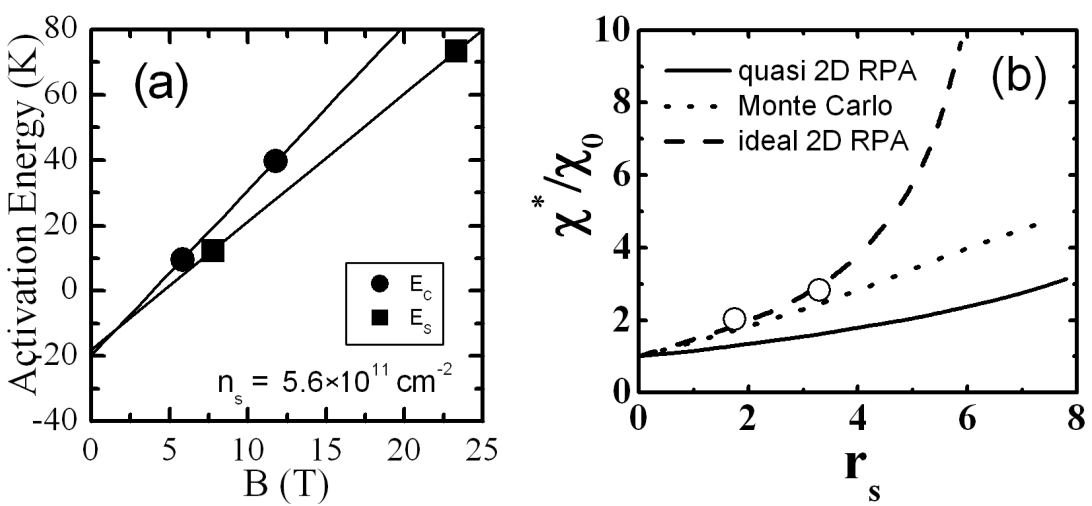

Fig. 2. The plots of activation energies in spin $\left(E_{\mathrm{s}}\right)$ and cyclotron $\left(E_{\mathrm{c}}\right)$ gaps vs. magnetic field (a). Dependence of relative spin susceptibility enhancement on interaction strength $r_{\mathrm{s}}(\mathrm{b})$.

is given by $R_{x x} \propto \exp \left(-\Delta E / 2 k_{\mathrm{B}} T\right)$. The solid lines in Fig. 1b represent the fits according to this formula. The obtained activation energies reflect directly spin gaps for odd filling factors and cyclotron gaps for even filling factors. Their dependence on magnetic field is presented in Fig. 2a. One can notice that all traces have a nonzero intercept for zero magnetic field. It is the consequence of the finite level width $(\Gamma)$ related to the presence of the mobility edges within each quantized Landau level. Thus the spin gap is given by: $E_{\mathrm{s}}=g^{*} \mu_{\mathrm{B}} B-\Gamma$, and cyclotron gap $E_{\mathrm{c}}=e B / m^{*}-g_{0} \mu_{\mathrm{B}} B-\Gamma$ (here $g_{0}$ states for bare $g$-factor being equal to $2.0[4])$. The inferred data on electron-electron interaction enhanced $g$-factor and effective mass are gathered in Table. We have put also the values of effective mass after Ref. [5] (from cyclotron resonance experiment). These are pure bare values according to Kohn's theorem [6]. One can notice that electron-electron 
interactions only slightly modify effective mass, whereas its influence onto $g$-factor is substantial. A similar observation on $g$-factor enhancement in GaN/AlGaN heterostructure was already reported in Ref. [7]. Authors measured the spin splitting of the Shubnikov-de Haas oscillations from the peak positions relying on the crude assumption that the Fermi level does not depend on magnetic field. Therefore, their result has rather a qualitative nature.

TABLE

The effective mass and $g$-factors for GaN/AlGaN heterostructures.

\begin{tabular}{c|c|c|c|c}
\hline \hline$n_{\mathrm{s}}$ & $r_{\mathrm{s}}$ & $m_{0}$ & $m^{*}$ & $g^{*}$ \\
\hline $2.1 \times 10^{12} \mathrm{~cm}^{-2}$ & 1.75 & 0.225 & 0.251 & 3.6 \\
$5.6 \times 10^{11} \mathrm{~cm}^{-2}$ & 3.30 & 0.214 & 0.210 & 5.8
\end{tabular}

Both quantities, $m^{*}$ and $g^{*}$, enter the expression for spin susceptibility, thus it is instructive to compare it with theoretical predictions. We have chosen quantum Monte Carlo results from Ref. [8] and calculations made in random phase approximation from Ref. [1]. In Fig. $2 \mathrm{~b}$ the relative spin susceptibility is depicted $\left(\chi^{*} / \chi_{0}=g^{*} m^{*} / g_{0} m_{0}\right)$. Here the experimental data coincide with an ideal twodimensional system model in random phase approximation (finite width of $2 \mathrm{D}$ channel neglected). Although the quantum Monte Carlo description is also not bad. The situation will be much clearer when still lower density samples became available. At the present stage it seems that electron-electron interactions influence mainly effective $g$-factor and it is responsible for enhanced spin susceptibility. Finally, the comment is needed about our way of evaluation of the spin susceptibility. Namely, it is usually deduced from critical field in parallel configuration when the resistivity saturates in a fully spin polarized state or from the Landau level coincidence measurements or at least from measurements of magnetization as a function of magnetic field. All these methods involve finite magnetic field, while one has to remember that true thermodynamical spin susceptibility is defined as a derivative of the magnetization over magnetic field in the limit of zero magnetic field. Our technique of obtaining spin susceptibility from the activated resistivity involves relatively higher magnetic fields. In this case one should expect even more pronounced nonlinearities of spin susceptibility with magnetic fields as predicted by the latest theory [9]. But fortunately such a nonlinear behavior should be visible in the magnetic field dependence of activation energies, which was not yet observed (Fig. 2). Thus we justify a posteriori our way of evaluation of the spin susceptibility.

In conclusion, we have deduced the electron-electron interaction enhanced effective mass and $g$-factor from the activated resistivity in the quantum Hall regime of $2 \mathrm{D}$ electron gas in $\mathrm{GaN} / \mathrm{AlGaN}$ heterostructures in the limit of interme- 
diate interaction strength. Further, the resulting spin susceptibility fits well with the ideal model of the $2 \mathrm{D}$ electron gas in random phase approximation.

\section{Acknowledgments}

The work has been partially supported by the "Transnational Access Specific Support Action" Program — contract no. RITA-CT-2003-505474 of the European Commission.

\section{References}

[1] Ying Zhang, S. Das Sarma, Phys. Rev. B 72, 075308 (2005).

[2] V.M. Pudalov, M.E. Gershenson, H. Kojima, in: Fundamental Problems of Mesoscopic Physics. Interaction and Decoherence, Eds. I.V. Lerner, B.L. Altshuler, Y. Gefen, NATO Sci. Series II, Mathematics, Physics and Chemistry, Vol. 154, Kluwer, New York 2004, p. 309.

[3] C. Skierbiszewski, K. Dybko, W. Knap, M. Siekacz, J. Łusakowski, W. Krupczyński, G. Nowak, M. Boćkowski, Z.R. Wasilewski, D. Maude, T. Suski, S. Porowski, Appl. Phys. Lett. 86, 102106 (2005).

[4] A. Wołoś, W. Jantsch, K. Dybko, Z. Wilamowski, C. Skierbiszewski, in: Proc. 28th Int. Conf. on the Physics of Semiconductors, Vienna 2006, Eds. W. Jantsch, F. Schaffler, AIP Conf. Proc. 893, 1313 (2007).

[5] S. Syed, J.B. Heroux, Y.J. Wang, M.J. Manfra, R.J. Molnar, H.L. Stormer, Appl. Phys. Lett. 83, 4553 (2003).

[6] W. Kohn, Phys. Rev. 123, 1242 (1961).

[7] K.S. Cho, Tsai-Yu Huang, Chao-Ping Huang, Yi-Hsing Chiu, C.-T. Liang, Y.F. Chen, J. Appl. Phys. 96, 7370 (2004).

[8] C. Attaccalite, S. Moroni, P. Gori-Giorgi, G.B. Bachelet, Phys. Rev. Lett. 88, 256601 (2002)

[9] Ying Zhang, S. Das Sarma, Phys. Rev. Lett. 96, 196602 (2006). 